\title{
Representing locational requirements using conventional decision tables: theory and illustration
}

\author{
Frank Witlox ${ }^{(1)}$ and Harry Timmermans ${ }^{(2)}$ \\ (1) Ghent University, Faculty of Sciences, Department of Geography, Krijgslaan 281 (S8), B-9000 \\ Gent, Belgium, Tel.: + 32 (0)9 26445 53, Fax: + 32 (0)9 26446 95, E-mail: frank.witlox@rug.ac.be. \\ (2) Eindhoven University of Technology, Department of Architecture, Building and Planning, Urban \\ Planning Group, Mail box 5.13, NL-5600 MB Eindhoven, The Netherlands, Tel.: + 314024722 \\ 74, Fax: + 314024758 82, E-mail: h.j.p.timmermans@bwk.tue.nl.
}

Keywords: location theory, decision tables, site selection, petrochemical industry

Paper resubmitted for possible publication in Geographical and Environmental Modelling.

[November 2000] 


\section{Introduction}

When faced with the problem to search for a feasible location, decision-makers have to consider the relevance of a wide variety of factors, including site conditions, investment considerations, and operating costs. In some cases, potential locations need to satisfy particular conditions, otherwise they will be rejected as a potential choice option. In other cases, decision-makers will have some ideal value in mind, and the suitability of a location will decrease as the actual value of a location deviates from this ideal point. Yet other factors will have an increasing or decreasing utility.

The challenge for the researcher is how to represent these locational requirements. A formal representation is required to build models of locational decision making to predict the behaviour of firms or assess locational policies. It is also a necessity to develop decision-support systems that help decision-makers to screen possible locations against their set of locational requirements. Finally, formal representations of locational requirements may be of help in analysing location factors and locational decision-making processes.

In the current paper, we will advocate the use of decision tables in this context. A conventional decision table (DT) is a tabular representation used to describe and analyse procedural decision situations, where the status of a number of conditions jointly determine the execution of a set of actions. DT's have a substantial history in other disciplines, such as computer science, but we are not aware of any publication in the leading journals in the spatial sciences. Also important to stress is that the present paper initially departs from a deterministic view on DTs. As such, adaptions in which, e.g., rough or fuzzy set theory is applied, are not explicitly dealt with (see, e.g., Witlox, 1998).

We will first introduce the concept of a decision table and discuss its properties. Then, we will illustrate the use of decision tables taking the locational requirements of the petro-chemical industry as an example. The sample and the method of knowledge acquisition will be outlined, and some of the results will be described. The article concludes by discussing some critical issues in the application of decision tables and some avenues of future research. 


\section{Decision tables: theory}

Decision tables were initially introduced over three decades ago, primarily as a method in software engineering for structuring computer programmes (McDaniel, 1970; Montalbano, 1974). It was found that, as a formalism, DTs were very well-suited to describe and analyse problems that contain procedural decision situations which are characterised by a set of influential conditions, the state of which determines the execution of a set of actions (CODASYL, 1982). Later on, several other important application domains such as manual decision-making, system analysis and design, representation of complex texts, verification of knowledge bases, and knowledge acquisition emerged (Vanthienen and Dries, 1992, 1994). In recent years, the potential of DTs as a conceptual modelling language for representing qualitative and complex knowledge have been investigated (Lucardie, 1994; Arentze et al., 1995). In respect to this latter point, both Vanthienen (1986) and Lucardie (1994) have developed specific software (e.g. PROLOGA95 and AKTS) for constructing decision tables. These DT engineering workbenches have created a significant added value to the use of the DT technique.

For a more extensive overview on the history of decision table development and applications, we refer to Wets (1998).

\subsection{Definition of a decision table}

A decision table (DT) is "a table that represents the exhaustive set of mutually exclusive conditional statements within a pre-specified problem area [my translation]" (Verhelst, 1980, p. 9). It displays the possible actions that a decision-maker can follow according to the outcome of a number of relevant conditions. The general structure of a DT is shown in Figure 1.

\section{Figure 1: The general structure of a decision table}

\begin{tabular}{|l||l|}
\hline Problem area & \multicolumn{2}{|l}{} \\
\hline CONDITION SET & CONDITION SPACE \\
\hline \hline ACTION SET & ACTION SPACE \\
\hline
\end{tabular}


Each DT is identified by the problem area it investigates (e.g. select suitable location). The table is split by a double line, both horizontally and vertically. The horizontal line divides the table in a condition part (above) and an action part (below). The vertical line divides the sets or stubs or subjects (left) from the spaces or entries or states (right). The result is four quadrants: condition set $\left[C_{\mathrm{i}}\right]$, action set $\left[A_{\mathrm{j}}\right]$, condition space $\left[\operatorname{SPACE}\left(C_{\mathrm{i}}\right)\right]$, and action space $\left[\operatorname{SPACE}\left(A_{\mathrm{j}}\right)\right]$.

The condition set consists of all the relevant conditions or attributes (inputs, premises or causes) that have an influence on the decision-making process. For instance, in respect to the choice of a business location, the set of relevant conditions consists of all attributes that influence the process of selecting a location site. More formally, a condition set is denoted as follows:

\author{
Notation 1: Condition set \\ $C_{\mathrm{i}}$ denotes a condition set with domain $C D_{\mathrm{i}}\left(i=1, \ldots, C_{\text {num }}\right)$ with $i$, the set of numerical indices \\ indicating the condition, and $C_{\text {num }}$, the number of conditions.
}

The condition space specifies all possible combinations of condition states of a condition. The number of possible condition states is unlimited, at least in theory. It could range from one to any desired number. However, the more condition states used, the more complex the decision table structure will be. More formally, the condition space and condition state are denoted as follows:

\title{
Notation 2: Condition space, condition state
}

For every $i=1, \ldots, C_{\text {num }}$ the condition space $\operatorname{SPACE}\left(C_{\mathrm{i}}\right)$ is denoted as the set containing the condition states $C S_{\mathrm{ik}}$, i.e. SPACE $\left(C_{\mathrm{i}}\right):=\left\{C S_{\mathrm{ik}} \mid \mathrm{k}=1, \ldots, n_{\mathrm{i}}\right\}$, where $n_{\mathrm{i}}$ is the number of condition states corresponding to condition $i$

Binary condition states (e.g. yes/no) are termed "limited-entry" conditions. Condition states that can account for more than two possible outcomes are termed "extended-entry" conditions. A DT that combines both limited and extended entries is termed a "mixed-entry" DT. Condition states that are 
irrelevant for a certain decision rule are marked with the so-called "don't care" entry (denoted: "-"). This "don't care" entry is equal to stating that all condition states are allowed in a disjunction.

The action set contains all the possible actions (outputs, conclusions or consequences) a decision-maker is able to take. This is to say that the action set points to the possible choice outcome if, for instance, an existing location with a number of specific characteristics is processed through the DT. DTs can account for as many actions as the decision-maker feels it necessary to introduce. The formal notation of an action set is given as follows:

\author{
Notation 3: Action set \\ $A_{\mathrm{j}}\left(j=1, \ldots, A_{\text {num }}\right)$ denotes an action set with $j$, the set of numerical indices indicating the \\ action, and $A_{\text {num }}$, the number of actions.
}

The action space contains the categorizations of all the possible action states of an action. The number of possible action states is also unlimited. However, frequently, DTs confine the number of action states (not number of actions) to three. These are "-", "x" and ".". The narrow line ("-") indicates that the particular action may not be executed, the cross-sign ("x") implies that the particular action must be followed, and a dot (".") signifies an undefined action state. More formally, action space and action state are denoted as follows:

\title{
Notation 4: Action space, action state
}

For every $j=1, \ldots, A_{\text {num }}$ the action space $\operatorname{SPACE}\left(A_{\mathrm{j}}\right)$ is defined as the set containing the action states $A S_{\mathrm{jk}}$, i.e. SPACE $\left(A_{\mathrm{j}}\right):=\left\{A S_{\mathrm{jk}} \mid \mathrm{k}=1, \ldots, n_{\mathrm{j}}\right\}$, where $n_{\mathrm{j}}$ is the number of action states corresponding to action $j$.

The strict separation between conditions and actions may not always be as simple as it might look. Some actions are bound to a condition, implying that they have to be executed before testing the condition, while others may already be executed after testing one or more conditions. Also, condition 
sets and action sets may themselves refer to other decision tables. For example, an action may involve a call to a procedure to perform some task, or a condition may be defined by another decision table. A decision table called in that way by another decision table is termed an action- or a condition-subtable. These subtables play an important role in structuring the decision problem and permit a more thorough and detailed analysis (see below).

Finally, any vertical linking of an element out of the condition space or categories with an element of the action space or categories produces a so-called logical rule [R]. This logical rule is in fact a simple conditional statement or an "if...then" decision rule.

On the basis of the above-stated definitions, a decision table is defined as follows (Reilley et al., 1987, pp. 196-197; Vanthienen en Dries, 1992; Lucardie, 1994):

\section{Definition 1: Decision table}

A conventional decision table is defined as a function DT that maps each possible combination of condition states to one (exhaustivity) and only one (exclusivity) action state, i.e. DT : SPACE $\left(C_{1}\right) \times \operatorname{SPACE}\left(C_{2}\right) \times \ldots \times \operatorname{SPACE}\left(C_{\text {num }}\right) \rightarrow \operatorname{SPACE}\left(A_{1}\right) \times \operatorname{SPACE}\left(A_{2}\right) \times \ldots \times$ $\operatorname{SPACE}\left(A_{\text {num }}\right)$

Apparently, in order to account for exhaustiveness and exclusiveness, the following two logical constraints must be fulfilled for each $C S_{\mathrm{i}}$ of a DT:

\section{Definition 2: Exhaustiveness}

(1) $\cup C S_{\mathrm{i}}=C D_{\mathrm{i}}$

\section{Definition 3: Exclusiveness}

(2) $\cap C S_{\mathrm{i}}=\varnothing$. 
The two logical constraints respectively employ the union and intersection operation of classical set theory. The first constraint states that the union of all condition states of condition $\left(C S_{\mathrm{i}}\right)$ should be equivalent to the domain of that condition $\left(C D_{\mathrm{i}}\right)$. The second constraint states that the intersection between different condition states of a condition should yield the empty set $(\varnothing)$. As such, $C S_{\mathrm{i}}$ must be disjunct.

Note that the view taken here on DTs is a deterministic one. Condition states and action states are not allowed to overlap. In other words, a strict ('crisp') set theory-based methodology of decision tables is followed. This feature is regarded to be both a strength as a weakness. The strength follows from the fact that the conventional DT obey the principle of dichotomy which states that a statement (or evaluation) is either true or false. Hence, a non-fuzzy decision-making process is assumed. It is also a weakness because a crisp (non-rough or non-fuzzy) DT is unable to account for imprecisions and vagueness. The introduction of rought sets (Pawlak et al., 1995) or fuzzy sets (Zadeh, 1965) could alleviate to a certain extent some of these drawbacks.

\subsection{Construction of a decision table}

Verhelst (1980, p. 23) distinguishes three methods to construct a decision table or decision table system: (i) a direct method on the basis of singular (simple) decision rules; (ii) a direct method on the basis of composite (complex) decision rules; and (iii) a search method. Although, these three methods are different on a number of factors (see below), they deal with comparable stages or steps when converting a decision problem into a DT. The main difference between them is the order in which these stages are treated and the way they are worked out. In sum, five stages may be distinguished (Vanthienen and Wets, 1994, p. 269):

(i) Definition of conditions, condition states, actions and action states for the specific choice problem;

(ii) Specification of the problem in terms of decision rules;

(iii) Construction of the DT on the basis of the decision rules;

(iv) Check for completeness, contradictions and correctness; 
In reality, the construction of decision tables is often not a linear process as the above procedure seems to suggest because of the complexity of the task. The different stages indicate a way to structure this task. In what follows, we elaborate on each of these five stages.

When building a DT, an essential and rather straightforward first step is the identification of the conditions and actions and their associated states that have an influence on the decision problem. In this respect, the three above-mentioned DT construction methods strongly resemble conventional quantitative or other qualitative approaches. Usually, the decision maker is asked to list or name all conditions that are deemed important in evaluating a choice alternative. These verbal records or protocols can be completed with conditions derived from other sources (e.g. literature survey). As such, a combination of condition-determining approaches is used that define the influential conditions both a priori and a posteriori to the decision maker's answers. Next, the condition states are defined. It is important to note that the decision maker is not limited to only two states. The decision maker is able to express as many states as he or she feels necessary in order to logically evaluate a certain condition. All attributes are evaluated in terms of their logical condition states; whether they are limited or extended entries is not important. This offers a greater flexibility to the researcher. Also, the use of (different) multiple response categories implies that conditions are allowed to interact (conceptual interaction). Linked with the aspect of condition categorisation is that also dependencies between conditions are taken into account (conditional relevance). As such, a condition like "distance" can be categorised into three, five, or, for that matter, any other number of different categories. Comparable to the condition and condition state specification, the possible actions and action states need to be determined. Again, this can be accomplished by asking the decision maker what possible actions he or she anticipates in considering the choice alternatives. The number of actions will depend on the nature of the choice problem, and is therefore not limited.

As a rule, conditions (or attributes) and actions have to be unambiguous, relevant and realistic to the decision-maker. All condition recurrences, as well as all complementary conditions, should be avoided. Conditions that depend upon other conditions should separately be treated, and ranked in 
such a way that dependent conditions follow independent conditions. In respect to the categorisation of the conditions, two important logical requirements must be fulfilled: exhaustivity (Definition 2) and exclusivity (Definition 3). Exhaustivity means that the DT must account for all possible states that a condition is able to take. The exclusivity requirement refers to the fact that each combination of condition states has to be included in one and only one column of the DT. A brief example will perhaps further clarify both logical requirements. Suppose, for an arbitrary chosen condition "distance" (d), the condition states are specified as follows: $d<100,100 \leq d \leq 150, d>150$. It is clear that this condition categorisation is exclusive because no single distance can at the same time fall in more than one category. In addition, the categorisation is also exhaustive because all possible distances are captured in either one of the three condition states. Therefore, an important property of using decision tables is that the condition states are mutually exclusive but jointly form an exhaustive set.

The second stage in the construction process concerns the specification of the decision rules. A decision rule is a logical vertical link between elements of the condition space (antecedent or premise) and action space (consequent or conclusion). It can be compared with a simple "if...then" decision rule. By this is meant: if faced with a number of conditions, then execute a certain action.

With respect to the three above DT construction methods, different decision rules or techniques are applied. In the first direct method, singular (or simple) "if...then" decision rules are advanced. This implies that each possible combination of condition states will lead to exactly one and only one action state or column of the table. The result is a totally unambiguous, but rather complex DT. To illustrate, if a location site is characterised by nine conditions, all defined in terms of only two condition states, this will result in $2^{9}$ (or 512) singular decision rules. Clearly, such a table is too complex to be interpreted. Note, however, the similarity with a full factorial design in stated preference modelling.

The second direct method deals with composite (or complex) "if...then" decision rules and is somewhat more difficult to understand. Composite rules are based on the combination of singular rules in such a way that in a column for a particular condition, its associated states are being 
contracted. Thus, singular rules are joined to form a composite rule. A brief example may clarify this somewhat further. Suppose that a DT consists of only two conditions $\left(C_{1}\right.$ and $\left.C_{2}\right)$ with associated condition states $\left(C S_{11}, C S_{12}\right)$ and $\left(C S_{21}, C S_{22}, C S_{23}\right)$. In a singular DT, this would result in six columns or six singular decision rules. Assume further that for $C S_{12}$, the condition states of $C_{2}$ become irrelevant. Thus, in the $C S_{12}$-column, $C S_{21}, C S_{22}$ and $C S_{23}$ may be combined in a single state, namely the so-called "don't care" entry (denoted: "-"). The result is a composite DT that has only four columns (three singular rules and one composite rule). Such grouping or contraction of condition states may cause a loss of overview, and with it, a loss of the simplicity needed to control the exclusivity and exhaustivity requirements of the DT. Therefore, composite rules are only used for problems that are relatively simple and easy to understand.

In the third approach, the search method, yet another technique is applied, which is called equivalence class or rule class. The idea was advanced by the Decision Table Task Group of CODASYL (1982). Equivalence class ruling denotes all simple decision rules that have identical or equivalent action states, regardless of the combination of the condition states. Note that in the functional classification theory, equivalence or rule class is termed functional equivalence. Therefore, decision rules are assumed functionally equivalent if they point to identical action states although they are based on different combinations of condition states.

\section{Table 1: Construction of an abstract multiple-hit DT using the search method}

\begin{tabular}{|c|c|c|c|c|c|c|c|c|c|c|c|c|}
\hline Condition $\mathrm{C}_{1}$ & \multicolumn{6}{|c|}{$\mathrm{a}$} & \multicolumn{6}{|c|}{ B } \\
\hline Condition $\mathrm{C}_{2}$ & \multicolumn{2}{|c|}{$\mathrm{C}$} & \multicolumn{2}{|c|}{$\mathrm{d}$} & \multicolumn{2}{|c|}{$\mathrm{e}$} & \multicolumn{2}{|c|}{$\mathrm{F}$} & \multicolumn{2}{|c|}{$\mathrm{g}$} & \multicolumn{2}{|c|}{$\mathrm{h}$} \\
\hline Condition $\mathrm{C}_{3}$ & $\mathrm{Y}$ & $\mathrm{N}$ & $\mathrm{Y}$ & $\mathrm{N}$ & $\mathrm{Y}$ & $\mathrm{N}$ & $\bar{Y}$ & $\mathrm{~N}$ & $\bar{Y}$ & $\mathrm{~N}$ & $\mathrm{Y}$ & $\mathrm{N}$ \\
\hline Action $\mathrm{A}_{1}$ & $\mathrm{x}$ & - & - & - & $\mathrm{x}$ & & & & & & & \\
\hline Action $\mathrm{A}_{2}$ & $\mathrm{x}$ & $\mathrm{x}$ & $\mathrm{x}$ & $\mathrm{x}$ & - & & & & & & & \\
\hline Action $\mathrm{A}_{3}$ & $\mathrm{x}$ & - & $\mathrm{x}$ & - & - & & & & & & & \\
\hline Action $\mathrm{A}_{4}$ & - & $\mathrm{x}$ & - & $\mathrm{x}$ & - & & & & & & & \\
\hline Decision rule & $\mathrm{R}_{1}$ & $\mathrm{R}_{2}$ & $\mathrm{R}_{3}$ & $\mathrm{R}_{4}$ & $\mathrm{R}_{5}$ & & & & & & & \\
\hline $\begin{array}{l}\text { Frame of } \\
\text { functional } \\
\text { equivalence }\end{array}$ & 1 & 2 & 3 & 2 & 4 & & & & & & & \\
\hline
\end{tabular}

Translated to the choice of business location, two location sites are assumed functionally equivalent if both locations, although having very different characteristics, can perform an equivalent function (i.e. site suitability) for an economic activity. To further illustrate the search method and the technique of functional equivalence in DTs, we refer to the abstract DT presented in Table 1. Suppose that an 
analyst, when constructing a DT, has come to a situation as depicted in Table 1. So far, five decision rules have been identified, and in this early stage of the construction process, it is clear that the decision rules $R_{2}$ and $R_{4}$ fall within the same frame of functional equivalence. This is because the two particular decision rules exhibit the same configuration of action states, although their associated condition states ( $\mathrm{acN}$ and $\mathrm{adN})$ are different. When such a frame of functional equivalence is detected, the analyst is interested in whether a statement like "A "no" for condition $C_{3}$ always implies that both actions $A_{2}$ and $A_{4}$ must be executed?" is true. There are two possible outcomes: either this statement is not true, in which case the analyst continues in further defining decision rules $R_{6}$, $R_{7}$, etc., until a new frame of functional equivalence is observed for which the same procedure is then followed; or, the statement is presumed true, in which case the analyst confronts the decision-maker with other combinations of conditions states of $C_{1}$ and $C_{2}$ ( $C_{3}$ still being equal to "no"), and examines whether action $A_{2}$ and $A_{4}$ still have to be executed. To reduce the complexity of the analyst's task, one is able to work statistically. For instance, the decision-maker is confronted with only $50 \%$ or $75 \%$ of all possible combinations of condition states of $C_{1}$ and $C_{2}$. If in all cases the result stays unchanged, one may take for granted that a "no" for $C_{3}$ automatically results in executing $A_{2}$ and $A_{4}$. The remainder of the analysis can then be concentrated on what the choice-maker will do if $C_{3}$ takes the condition state "yes". A consequence of functional equivalence is that the DT will not lead to a unique choice, but presents a number of functionally equal choices. It is important to note here that DT's satisfy the necessary conditions for reconstructing decision rules defining functional equivalence.

With respect to the definition of decision rules, it is also useful to refer to the existence of what it termed the ELSE rule (Verhelst, 1980, p. 90; CODASYL, 1982, p. 3.41). The ELSE rule is a rule that is executed when none of the other decision rules in a DT are satisfied. It may be viewed as a kind of "catch-all" when all other rules fail. Usually, the ELSE rule is placed as the last decision rule in a DT. The application of the ELSE rule brings with it two important drawbacks: (i) the control for completeness is lost, and (ii) the DT becomes less manageable and interpretable as several decisions are put together in one single column. Consequently, the use of the ELSE rule should be avoided.

With respect to decision rule induction, a system called LERS, developed by Pawlak et al. (1995), should be mentioned which is based on rough set theory. One of the arguments that could be 
used against an approach based on conventional DT is that a too deterministic point of view is taken. In LERS, rules may be induced using a machine learning approach or a knowledge acquisition learning approach. If the first option is chosen, the system induces a minimum discriminant decription for each class. Such a minimum discriminant description tries to cover all the positive instances from a label and not the negative ones. If the second option is selected, the system will induce in minimal form each rule that can be induced from the set of instances. An interesting feature is that the system is able to generate a set of certain rules (they are always true given the data) and a set of possible rules. Hence, rough set theory extents the basis idea that decision rules are induced on a mere deterministic basis.

The third stage deals with the actual construction of the DT on the basis of the defined decision rules. The ranking of conditions and actions in the table is not subject to a strict hierarchy. However, a certain logic in the condition order should help in the task of interpreting and optimising the DT (see also stage 5). Usually, dominant or so-called veto conditions are put at the top of the table because of their non-compensatory character. Also, in view of optimisation (in the present context: minimize the total number of decision rules), it is better to list conditions which have fewer condition states before conditions with relatively more condition states. Similar, conditions which are deemed more important to the choice problem need to be followed by the more impractical (or theoretical) conditions. After all, it is less plausible or likely that a decision-maker will disregard an attribute which seems to have a greater bearing upon the choice problem than that he or she will ignore a more impractical but theoretically possible influential attribute. Usually, conditions appear only once in the DT. By being able to organise and rearrange conditions and condition states in a DT, it is clear that the DT formalism supports the systematic account of conditional relevance of attributes and conceptual interactions between attributes. Both mechanisms underlie the concept of functional equivalence.

The fourth stage examines the DT with regard to the requirements of completeness, contradiction and correctness. Here, an automatic check is presented to detect certain specification errors. First, the check for completeness verifies whether the table contains decision rules which have empty action states. In other words, an incomplete DT is a table in which decision rules are missing. If 
this is observed, it can be rectified. Second, the DT is controlled for contradictions. A contradiction occurs if, leaving aside the ELSE rule, two action states which exclude one another must be executed at the same time. Such a contradiction usually follows from a bad specification of the condition states. Third, if a DT is complete and no decision rules are contradictory, this still does not mean that the decision rules are correct. The correctness of a decision rule depends on the choice-maker's interpretation. As a result to the exclusive and exhaustive character of DTs, all potential condition states are combined to form action states, including those combinations of condition states that result in unwanted, unrealistic and overlooked action states. In this stage of DT construction, these action states may be spotted and defined so that the DT will produce only valid decision rules. In practice, this comes down to investigating whether every individual decision rule is correct.

The fifth and final stage deals with the simplification, optimisation and depiction of the DT. Optimisation in this context refers to the minimisation of the number of decision rules in a DT. In other words, redundancy in the decision rules should be avoided. There are several ways to optimise the lay-out and execution time of a DT. Table contraction is one possibility, row order optimisation another (Vanthienen and Dries, 1994, p. 227).

Table contraction (or rule collapsing) refers to the minimisation of the number of columns for a given condition order by combining (groups of) columns which lead to the same action configuration. In other words, the number of columns is reduced by contracting singular to composite decision rules. Table contraction should always be preceded by the check for completeness, contradictions and correctness. The reason is that once the table has been contracted, it is difficult to uncover decision rule errors. Row order optimisation opens the possibility of decreasing the number of (contracted) columns by changing the order in which the conditions are noted in the table. For a table with $n$ conditions, this implies a choice between $n$ ! alternative condition orders. Some rankings, however, might not be feasible due to previous constraints (e.g. condition dependencies). A third way to optimise and structure a DT is by using subtables. A subtable may be defined as a decision table wherein actions function as conditions in an upper-level decision table. Subtables may themselves be subject to other subtables. Figure 2 shows a simple abstract example of a three-level decision table system with subtables. 


\section{Figure 2: The structure of a three-level DT with subtables}
LEVEL 3
LEVEL 2
LEVEL 1

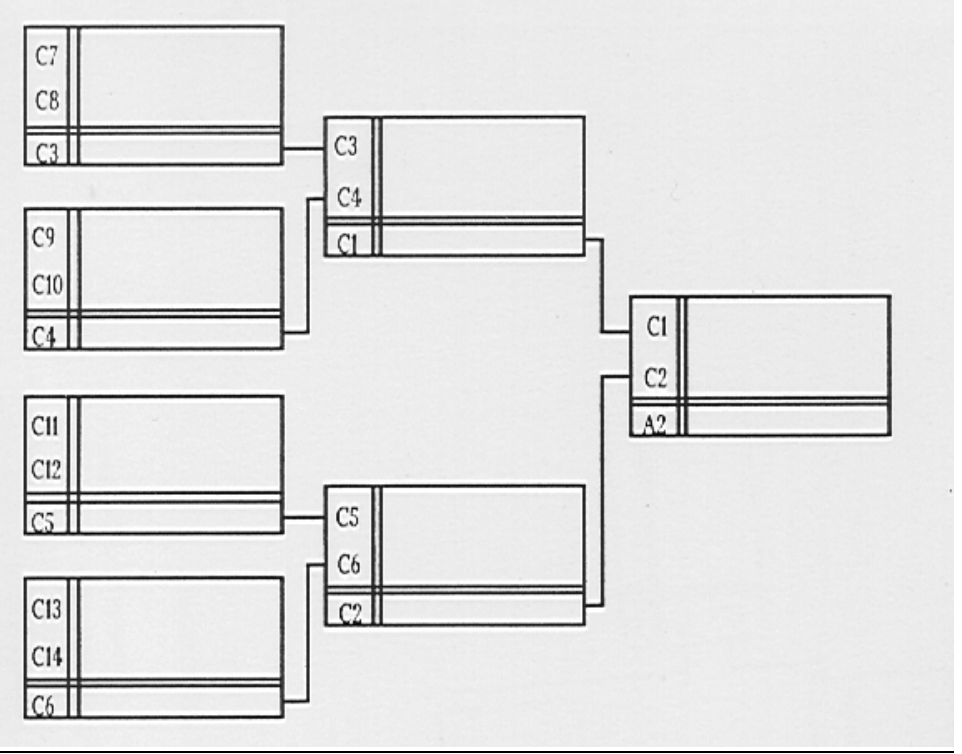

Source: Lucardie, 1988, p. 74.

In Figure 2 on the highest level (level 1$)$ only two conditions $\left(C_{1}\right.$ and $\left.C_{2}\right)$ explain the action state of

$A_{2}$. It can, however, be noted that both $C_{1}$ and $C_{2}$ are subject to the outcome of the subtables defined on the second level, and also subject to the results of the subtables specified on the third level. Therefore, defining $C_{7}, C_{8}, C_{9}$ and $C_{10}$ results in the specification of both $C_{3}$ and $C_{4}$ which in turn defines the condition state of $C_{1}$. Thus, $C_{1}$ is defined as a combination of four other condition states $\left(C S_{7}, C S_{8}, C S_{9}\right.$ and $\left.C S_{10}\right)$. In a similar way, $C_{2}$ is also defined as a combination of four condition states $\left(C S_{11}, C S_{12}, C S_{13}\right.$ and $\left.C S_{14}\right)$. Note that, in this simple example, the conditions $C_{3}, C_{4}, C_{5}$ and $C_{6}$ do not have to be specified because they are determined by the outcome of the two subtables on the third level. This property of generating knowledge from lower level condition state information is an important advantage of using a DT system. An additional characteristic of using subtables is that the concepts defining the conditions on higher levels may be more abstract than those on lower levels. Or, the higher (lower) the level in a DT, the more abstract (concrete) the concepts may be defined. Usually, this property entails that a condition relating to an abstract concept may be further specified through the use a condition-subtable. Conditions in a condition-subtable may in turn be further worked out in a subtable at lower level and so on. In many applications, DTs are connected through 
such condition-subtable links giving rise to an entire hierarchical system of decision tables. The highest level table specifies in abstract terms the decision rules in a problem domain. Subsequently, a system of subtables elaborates in more concrete terms these abstract concepts. The conditions used at the end points of the hierarchy should be defined in such a way that they can be relationally matched with the observable attributes (Arentze et al., 1996, p. 8).

\section{Decision tables: illustration}

To illustrate the use of DTs in the field of locational decision-making, this section will report the results of a case study. In particular, the chemical and petrochemical industry located in the port of Antwerp is taken as an example. There are several reasons why the chemical and petrochemical industry can be considered an appropriate case study. First of all, the industry has, in comparison, received only scant attention in the economic and regional location theory, despite its importance to the national and regional economy. Second, the capital-intensive nature of the industry makes it a sector that is very locational sensitive. Consequently, the location decision of a (petro)chemical complex is taken with great care, which gives an indication of the importance the sector attaches to the selection of a satisfactory, suitable site. Moreover, once a commitment for a certain location site has been made, it is generally considered irreversible, and therefore every factor influencing the profitability of the plant should be evaluated carefully. Third and finally, the petrochemical industry, like the chemical industry in general, is essentially a supplier of intermediate products to other industries. This specific role has enabled it to become a vital element of economic growth, and also emphasizes the importance of functional linkages.

The grounds to select the port of Antwerp as potential place of business are twofold. First, the present structure and development of the port is almost completely due to the increasing presence of the (petro)chemical industry. Consequently, today, Antwerp is considered as one of the largest chemical and petrochemical complexes in the world. Furthermore, in Western Europe, it is one of the oldest (Molle and Wever, 1984, p. 128). Obviously, this fact highlights Antwerp's apparent ability to attract chemical and petrochemical companies to its port. Second, the current, 
phased development of the Left Bank makes the port of Antwerp unique in its ability to continue to offer to potential interested companies large areas of industrial sites. As a result, several new (petro)chemical companies have recently decided in favour of Antwerp for establishing their operations.

In what follows, we will briefly outline the sample used, the method of knowledge acquisition to elicit the relevant locational requirements and discuss some of the results.

\subsection{General description of the sample}

In order to be able to represent the locational requirements of the (petro)chemical industry by means of DTs, a series of knowledge elicitation sessions were carried out. At the start of the data collection process in October 1996, the total number of chemical and petrochemical manufacturing companies located in the Port of Antwerp was equal to 26 (Havenbedrijf Antwerpen, 1996). Although, this number may seem small, the industry's combined economic impact on the Port of Antwerp is astonishing. The advantage of working with a rather small, but yet very important target population, is that no prior selection of a subgroup is necessary. However, it also implies that, in order to be representative, a high response rate is essential.

In October 1996, a personalized letter was mailed to each of the 26 enterprises with the request to grant an interview with the company's highest management executive on the subject of plant location decisions. In response to this letter, 14 companies answered initially, while, as a result of a short (i.e. three week period) telephone follow-up, an additional 9 companies could be persuaded to participate. Thus, the total response rate was equal to 23 companies (or $88 \%$ ). Given the relatively small population size (26 firms), the response rate is a exceptionally good. However, viewed in terms of sampling, it is a well-known that the smaller the size of the population, the higher the imposed demands on the resulting response rate. The three companies that did not respond and could be neglected in terms of importance were nevertheless asked to supply (by fax) some basic information regarding their operations in Antwerp. Eventually, two companies complied with this request. Due to the guaranteed anonymity of the participating companies, no firm-specific results will be reported. 
Given the difficulty of the task and the confidentiality of the issue, it is important that the experts are very well informed individuals, who are closely involved in final corporate decisionmaking and long-term strategic investment planning. The expert should also have a high level of interaction with the problem being analysed. Moreover, because sometimes the location decision was made some time ago or taken by the foreign parent company, the expert should preferably be a long-term serving employee, having a clear notion of how the initial corporate site was selected and which factors had played an important role. In view of these stringent expert requirements, only a high ranking executive, preferably the company's plant manager or the managing director, is able to fulfil the role of an "eligible" expert.

In 18 of the 23 interviews, the interviewee was indeed (one of) the highest ranking management decision-maker(s) of the company, while in the other four cases, the interviewee was either a high ranking assistant to that decision-maker or a senior middle staff member. In total, the group of high ranking management decision-makers consisted of 9 plant or operations managers, 7 (managing) directors, 1 general manager, and 1 member of the board. To the group of high ranking assistants or senior middle staff members belonged 1 finance and control manager, 1 area attorney, 2 heads of public relations, and 1 site service manager.

\subsection{Knowledge acquisition}

The interviews all took place during the period October 1996 to February 1997, with a peak in the months November (13 interviews) and December (6 interviews). On average, the actual interview lasted one hour to an hour and a half. All interviews were tape recorded (with the permission of the interviewee), and subsequently transcribed (usually the same day). Although, the process of interviewing can sometimes be tedious and may place great demands on both the expert as well as the interviewer, it requires little equipment, is highly flexible, and can yield a considerable amount of information in a relatively short time.

It is also important to note that all interviews proceeded in much the same way. First, the basic objective of the research was made clear to the expert. Next, the concept of a decision table (DT) as a formalism to represent data was explained. The expert was shown an example of a DT, 
and asked (if possible) to think and express his or her information in terms of "if...then" decision rule structures. As pointed out by Vanthienen (1986) and Arentze et al. $(1995,1996)$, this approach of data collection or elicitation, in which, from the start the expert is confronted with the notion of DTs, offers some significant advantages, especially in the case when the decision information is complex.

At least three reasons can be mentioned in support of such an approach. First, the communicative properties offered by the DT technique makes it an ideal formalism for representing complex information and sets of decision rules in such a way that is intelligible and clear to people. Note that, in this respect, one of the main advantages of a DT structure is its capacity of data representation. Second, DTs offer experts the possibility to specify and verify the correctness of their supplied information represented in the rules of the DT. Consequently, the model's accuracy can be tested in a systematic way by checking each conditional statement (i.e. decision rule) of the DT separately. Third and finally, given that DTs allow for the use of subDTs, they support a hierarchical structuring of information that does not only provide a form of modularity, but also helps to keep an overview of the decision problem under investigation. As such, a so-called "topdown" decision-making approach is followed in which one starts with an abstract (head) DT that is then further worked out in a series of more concrete subDTs. This approach also seems to correspond with the way people tend to transfer their information to the interviewer (Arentze et al., 1995, p. 240), and also concurs with the way in which a location decision is usually made .

The actual interview or knowledge-elicitation technique, which was used to elicit the decision information from the experts, can be termed a combination of an unstructured (informal) and a structured (guided) interview approach (Turban, 1995). In the unstructured, first part of the interview, the interviewee was asked to freely "think-aloud" on the subject of site selection (e.g. Which factors play a role in site selection?; Given a certain location factor, how is this factor assessed and what is its influence on locational decision-making?). The aim of this approach was to identify these location factors (i.e. conditions) that first came into the expert's mind, and more importantly, to know how these factors should be interpreted and which evaluation criteria are used. As such, if the interviewee stated that a location factor like "site accessibility" is important in 
selecting a suitable site, it is essential to know what is meant by that particular condition. For example, it may refer to evaluating the site's accessibility in general, or as was often the case, refer to the site's accessibility in respect to the supply of raw materials, the transportation of finished goods, or the transfer of the work force to and from the industrial site? Obviously, interpretations and relative importance of factors differed across the sample. However, usually a close link with the organizational and production-related aspects of the economic activity could be observed. This fact again emphasizes the importance to take account of the so-called "context-dependent" nature of location factors. To illustrate, for an air-splitting company, using air (i.e. a ubiquity) as principal feedstock, will require different site accessibility requirements (if any) with respect to feedstock supply from those put forward for an oil refinery which relies heavily on the overseas transport of crude oil and pipeline connections for the supply of its feedstock.

Another interesting point that could be deduced from the unstructured part of the interview, is that abstract location factors are usually not evaluated as such, but are first more concretely defined and then assessed. As a result, generic location factors such as "transport", "labour", "utilities" etc. are mentioned more than once in the decision-making process, but at different levels of importance in the site selection process and with varying interpretations. For instance, the location factor "transport" is first (on the highest level) interpreted and evaluated in terms of the availability of on-site "transport infrastructure". Conditional upon this evaluation, potential sites may either be rejected simply because their existing transport infrastructure is totally inadequate, or further assessed in terms of, e.g., the level of additional "transport investments" needed. Again dependent upon this second evaluation, a further assessment may be required of the site's "transport costs" and "accessibility". The basic idea behind this thought process is that site selection makers are not interested in how good or bad a potential site scores on, say, accessibility for personnel, if certain higher priority requirements concerning the general transport infrastructure are not met by the site. As such, a hierarchical decision-making process can be distinguished in which: first, on the highest level, a number of elementary site conditions are being evaluated, then a number of investment considerations, and finally, a number of operating considerations.

Apart from stressing the activity-specific nature of location factors, the individual 
influences of location factors on the site selection problem (e.g. veto-dimension, trade-off dimension) and the existence of internal dependencies between factors (i.e. conditional relevance, conceptual interaction) should also be examined. In this respect, if a certain location factor is evaluated as (un)satisfactory, then how does this evaluation effect the overall site selection process, and also the subsequent evaluation of other location factors? In addition, it is also important to know which evaluation criteria the expert uses to assess different location factors.

In the ensuing, structured part of the interview, the expert was asked to react to a check list of different location factors, and was also more closely guided through a series of questions about the subject of site selection. The check list used was compiled through a review of the relevant existing literature on the subject of chemical and petrochemical plantsite selection (e.g. Chapman, 1991). Among the additional questions posed, a number of so-called control questions were asked to eliminate certain basic inconsistencies. For instance, if the expert stated that the general labour market conditions are evaluated as very good, but later in the interview, it is found that the recruitment of suitable workforce is very difficult, this contradiction will have to be rectified. Having identified the principal factors (and their associated evaluation states) that are important in industrial site selection - i.e. the so-called domain layer of model development (Arentze et al., 1995) - the experts were urged (there where possible) to make "if...then" like statements or decision rules (i.e. the inferential layer) expressing their decision-making process.

The issue of actor heterogeneity in the actual organizational characteristics and spatial production requirements of different types of economic activity may cause a modelling problem in that different actors pursue different objectives. Hence, no two companies are identical, nor do they have complete similar locational preferences and strategies, not even if they are active in the same industry or branch of industry. The result is a diversity of actor profiles, demanding different locational requirements. The issue of actor heterogeneity can be easily illustrated. In our sample, the number of people employed by a single company ranges from 6 to 3,597, the occupied surface area extends from 0.23 ha to 595 ha, the annual production capacity varies from 5,000 to $8,400,000$ tons per year, and the total investment ranges from BEF 19 to 119,663 million. It is evident that, depending on the specific company, different location factors with varying intensity may come into 
play when selecting a suitable site.

The difficulty of dealing with activity-type heterogeneity implies finding a solution to the problem of how to model the activity-specific nature of location factors. Due to the variations in economic activity types in the sample, the (in)significance of location factors is subject to changes. The only appropriate solution to this important issue is to represent and incorporate the variation in activity types in the selected modelling approach. It is argued here that decision tables, as a modelling formalism, are capable of offering such a means.

\subsection{Some results}

According to the results of our interviews, a large number of location factors or attributes turned out to be relevant when selecting a suitable location site for a (petro)chemical industry. The decision-maker's site selection process also exhibited a typical hierarchical or nested structure, implying that the overall site selection problem is tackled by subdividing it into a series of less complicated subproblems.

Generalising over the total number of experts, three main site selection considerations could be distinguished. These are, in descending order of importance, (i) the overall site conditions, (ii) the investment considerations, and (iii) the operation considerations. Following this "top-down" approach, experts first evaluate a number of basic site conditions. These factors mainly relate to the geographical location, the acquisition conditions, the available on-site transport infrastructure, and the available on-site utilities. In second place, the investment considerations are assessed. The idea is that if the site conditions are not fully satisfactory, perhaps one could compensate for this inadequacy by making additional investments. However, there could be no way to compensate for sites evaluated as completely unsatisfactory (inferior). In that case, the site is rejected. The investment considerations refer to transport investments, utility investments, real estate considerations, and the level of government intervention. If the site conditions and the investment considerations have both been evaluated, the costs of operating the site will also have to be assessed. It involves evaluating the site accessibility, agglomeration economies, labour market, and utility costs. 
Through the use of an hierarchy of DTs these nested locational requirements can be made explicit. At the top level would be a head DT and linked to it, a series of subDTs each representing a subproblem. In our example, Table 2 depicts the contracted head DT (split in two parts, to fit the size of the page). It evaluates the site suitability of a harbour location for a (petro)chemical industry. In Table 2, three so-called INUS ('Insufficient but Necessary part of a conjunct set which is Unnecessary but Sufficient for the result')-conditions (i.e. a condition which is on its own insufficient, but within a conjunction indispensable) are presented which play a crucial role (on the highest decision level) in determining the degree of locational site suitability: $C_{1}$ : ${ }^{\wedge}$ Site conditions, $C_{2}:{ }^{\wedge}$ Investment considerations and, $C_{3}:{ }^{\wedge}$ Operating considerations. Note that each condition is defined using three condition states, which combined, results in $27\left(3^{3}\right)$ different decision rules (i.e. an expanded table). However, as a result of the non-compensatory character of the third condition state of $C_{1}$ and the apparent conditional relevance in the condition states of $C_{3}$, the total number of decision rules in the contracted table is equal to 15 . Of these 15 rules, several functionally equivalent rules (i.e. rules leading to identical action states, although having different condition state configurations) can be noted. Note further that Table 2 has only one action with five different action states. The action states express different degrees of site suitability, ranging from "excellent" (rule $R_{1}$ ) to "bad" (rules $R_{14}$ and $R_{15}$ ), dependent on the outcome of the condition set.

Table 2: Head DT evaluating the site suitability of a harbour location for a petro-chemical industry

\begin{tabular}{|l||c|c|c|c|c|c|c|}
\hline \multicolumn{1}{|l||}{ 1. C1 Site conditions } & \multicolumn{5}{c|}{ superior } \\
\cline { 2 - 9 } & \multicolumn{3}{|c|}{ 2. C2 Investment considerations } & \multicolumn{3}{|c|}{ good } & \multicolumn{2}{c|}{ about average } & \multicolumn{2}{c|}{ bad } \\
\hline 3. C3 Operating considerations & good & medium & bad & good or medium & bad & good & medium or bad \\
\hline \hline 1. A1 Excellent & $\mathrm{x}$ &. &. &. &. &. &. \\
\hline 2. A2 Above average &. & $\mathrm{x}$ &. & $\mathrm{x}$ &. &. &. \\
\hline 3. A3 Average &. &. & $\mathrm{x}$ &. & $\mathrm{x}$ & $\mathrm{x}$ &. \\
\hline 4. A4 Below average &. &. &. &. &. &. & $\mathrm{x}$ \\
\hline 5. A5 Bad &. &. &. &. &. &. &. \\
\hline
\end{tabular}




\begin{tabular}{|c|c|c|c|c|c|c|c|c|}
\hline \multirow{3}{*}{$\begin{array}{|ll|}\text { 1. } & \mathrm{C} 1 \wedge \text { Site conditions } \\
\text { 2. } & \mathrm{C} 2 \wedge \text { Investment considerations } \\
\text { 3. } & \mathrm{C} 3 \wedge \text { Operating considerations } \\
\end{array}$} & \multicolumn{7}{|c|}{ moderate } & \multirow{3}{*}{\begin{tabular}{|c|} 
inferior \\
- \\
-
\end{tabular}} \\
\hline & \multicolumn{2}{|l|}{ good } & \multicolumn{2}{|c|}{ about average } & \multicolumn{3}{|c|}{ bad } & \\
\hline & good or medium & bad & good & medium or bad & good & medium & bad & \\
\hline 1. A1 Excellent & . & . & . & . & . & . & . & . \\
\hline 2. A2 Above average & $\mathrm{x}$ & . & . & . & . & . & . & . \\
\hline 3. A3 Average & . & $\mathrm{x}$ & $\mathrm{x}$ & . & $\mathrm{x}$ & . & . & . \\
\hline 4. A4 Below average & . & . & . & $\mathrm{x}$ & . & $\mathrm{x}$ & . & . \\
\hline 5. A5 Bad & . & . & . & . & . & . & $\mathrm{x}$ & $\mathrm{x}$ \\
\hline
\end{tabular}

Each of the abstract conditions in Table 2 is further specified through a system of subDTs (denoted by "^"). Table 3 is an example of such a subtable. It represents the site conditions of a potential location. Given that one of the most important considerations in selecting a site is the assessment of the site itself with its specific characteristics, Table 3 makes an evaluation of the availability of a number of necessary production requirements (e.g., transportation facilities, public and firm-specific utilities, acquisition conditions, etc.) and an appraisal of certain basic environmental factors (e.g., geographical location, climate, living conditions, governmental regulations, etc.). Clearly, if a potential site is unable to meet the basic requirements demanded by an economic activity, it is considered totally unsuitable for locating that particular business. In other words, compensations for a less than adequate site are (at this stage of the site selection analysis) not allowed. Given the strong discriminating nature of the condition "^Site conditions", it is placed at the top of the head DT. It also implies that this condition will be evaluated first which, in fact, concurs with the way in which decision-makers tend to think when selecting sites: i.e. first, the general conditions of a site are evaluated, and conditional upon this evaluation, a further assessment is made. If the general site conditions are inferior, then that particular site is eliminated from further evaluation.

In the head DT (Table 2), condition $C_{1}$, representing "^Site conditions", is assessed using three condition states or categorizations: "superior", "moderate" and "inferior". As noted above, all "inferior" assessed sites are automatically rejected (i.e. a non-compensatory dimension) since they result in a "bad" site suitability evaluation (rule $R_{15}$ in Table 2). The actual evaluation outcome of $C_{1}$ is itself determined at a lower decision level by way of a system of four subDTs (see also Figure 
1). The condition subDT "^Site conditions" is depicted in Table 3. In Table 3, the condition "^Site conditions" is specified using four conditions, which are all subsequently defined by means of other subDTs. Note that the actions of Table 3 are identical (or have to correspond) to the condition states of the condition "^Site conditions" in the head DT. Again, non-compensatory and compensatory rules are used interchangeably. For instance, it can be noted that when condition $C_{1}$ "Geographical location" is evaluated as being "unsatisfactory", the overall site conditions are assumed "inferior" (i.e. action $A_{3}$ ). On the other hand, if $C_{1}$ and $C_{2}$ have been evaluated as "satisfactory" and "Ok", a "good" transport infrastructure (i.e. $C S_{31}$ ) combined with "lacking" utilities (i.e. $C S_{42}$ ) is functionally equivalent to a "medium" transport infrastructure (i.e. $C S_{32}$ ) with "adequate" utilities (i.e. $C S_{41}$ ). In both cases, the result is a "moderate" (i.e. action $A_{2}$ ) evaluation of the site conditions.

Table 3: Site conditions $\left(1^{\text {st }}\right.$ level contracted condition subDT $)$

\begin{tabular}{|c|c|c|c|c|c|c|c|c|c|}
\hline 1. $\mathrm{C} 1{ }^{\wedge}$ Geographical location & \multicolumn{8}{|c|}{ satisfactory } & \multirow{4}{*}{\begin{tabular}{|c|} 
unsatisfactory \\
- \\
- \\
-
\end{tabular}} \\
\hline 2. $\mathrm{C} 2 \wedge$ Acquisition conditions & \multicolumn{5}{|c|}{$\mathrm{Ok}$} & \multicolumn{3}{|c|}{ not Ok } & \\
\hline 3. $\mathrm{C} 3^{\wedge}$ Transport infrastructure & \multicolumn{2}{|c|}{ good } & \multicolumn{2}{|c|}{ medium } & \multirow{2}{*}{\begin{tabular}{|c|} 
bad \\
-
\end{tabular}} & \multicolumn{2}{|c|}{ good } & \multirow{2}{*}{ medium or bad } & \\
\hline 4. $\mathrm{C} 4 \cap$ Availability of utilities & adequate & lacking & adequate & lacking & & adequate & lacking & & \\
\hline 1. A1 Superior & $\mathrm{x}$ & . & . & . & . & . & . & . & . \\
\hline 2. A2 Moderate & . & $\mathrm{x}$ & $\mathrm{x}$ & . & . & $\mathrm{x}$ & . & . & . \\
\hline 3. A3 Inferior & . & . & . & $\mathrm{x}$ & $\mathrm{x}$ & . & $\mathrm{x}$ & $\mathrm{x}$ & $\mathrm{x}$ \\
\hline
\end{tabular}

It goes without saying that similar tables and subtables were constructed for the other decision influential factors. In total, no less than 91 DTs were constructed: i.e. one head decision table and, linked to it, a hierarchy of $90 \operatorname{sub}($ sub...)tables (see Witlox, 1998).

The hierarchical DT structure implies that abstract location factors defined in the head DT are further worked out by means of lower level, more concretely defined subtables whereby these subtables are themselves further specified by other subtables, and so on. Hence, abstract factors are not evaluated as such, but are first defined in terms of more concrete factors. This procedure also has the advantage that in representing location requirements all relevant elements (abstract or not) can be considered. The approach as such is not limited to a certain number of location influencing factors. 


\subsection{Properties of decision tables}

A useful property of DTs is that the direct, vertical linking of condition categories and action categories produces a decision rule which is accomplished without having to make underlying or intermediate measurements of the different condition states. These logical decision heuristics are then represented or visualised in a human-oriented table structure which makes the DT a suitable instrument to model difficult choice procedures without running the risk of losing control or oversight. The decision rules also exhibit a logical structure of a disjunction of conjoint terms, hence allowing to take account of internal related conditions. An additional advantage of DTs is that the formalism offers some means to partition a complex problem into a hierarchical structure of subtables, each of which can be considered a subproblem of its own. As such, the use of subtables enables one to divide a complex choice problem into smaller, more manageable problems.

Second, the definition of conditions, actions, condition states and action states can both be accomplished on an a priori and a posteriori basis. There are no restrictions imposed on the categorisation of the condition and action states. However, more important, the automated and computer supported DT construction offers a number of advantages which may avoid specification errors in the definition of these choice influencing attributes. In this respect, the verification capacity of software programmes (such as AKTS and PROLOGA) that help construct DTs can automatically check the table for the existence of (i) incompleteness and (ii) inconsistencies due to contradictions and redundancy in the problem specification. It is worthwhile to note that DTs imply a logical completeness which entails that "not a single action is unreachable and each and every possible combination of conditions leads to at least one action". An action (condition) is presumed unreachable if it is present in the DT but no conditions (actions) for it have been specified. This omission problem has been termed the "missing rule" problem by Nguyen et al. (1985, p. 376) which means that the DT specifies a particular decision rule for which knowledge is missing in order to match conditions with actions. Thus, logical incompleteness may occur as a result of unused or unreachable condition and action states, or because of missing input. An inconsistency may arise if, ignoring the ELSE rule, two action states which exclude one another must be executed at the same time. In other words, when 
identical combinations of condition states result in different action states an inconsistency is found. This form of inconsistency may be termed a contradiction (or conflict, ambiguity) and can easily be spotted in the DT. Preece and Shinghal (1994), Vanthienen and Wets (1994, p. 267) and Vanthienen et al. (1995) mention other forms of decision rule inconsistency: e.g. circular rules and redundant rules. Circular (or cyclical) rules are rules that somehow refer to themselves, either directly or indirectly via one or more other rules. Redundant rules are rules that point to identical conditionaction combinations. Clearly, neither of these inconsistencies will lead to errors during the consultation of the DT. However, both factors could seriously detract from the decision table's timeefficiency in producing a correct matching result. A somewhat more subtle form of inconsistency which is closely related to using a DT system is the problem of subsumption. Subsumption is linked to the use of subtables. It occurs if two sets of conditions refer to the same action, even though one of the sets is a subset of the other.

Third, the conditions and condition states are not weighed. As such, a decision rule is not based on some form of algebraic decision rule which might entail a compensatory decision-making model. Instead, in a DT, a combination of compensatory and non-compensatory rules can be used. On the one hand, a DT allows for compensatory decision-making because when a first condition is evaluated as unfavourable and it is not rejection-inducing, it can be compensated by the satisfactory result of another condition. On the other hand, the so-called dominant or veto conditions, usually placed at the top of the table, which induce the rejection of the alternative if they are not satisfied, could be viewed as non-compensatory. Different approaches can be followed which try to deal with the issue of compensatory versus non-compensatory decision rules. In a number of mathematical, utility-based models, these features can be built in. An alternative approach could be the use of a payoff matrix in which all trade-offs can be quantified and whereby non-compensatory trade-offs are given a score of negative infinity. Although these approaches seem useful, they do not offer the same, appealing features DTs do. For one, asking respondents to make exact quantifications of different trade-offs is not that simple. Moreover, the issue of dealing with internal dependencies between factors and the ability to construct disjunctions of conjoint decision rules is also not clear in an approach based on utility-modelling or pay-off matrixes. 
Thus, the decision table formalism seems to provide an attractive framework for representing locational requirements. However, an important potential drawback of the DT approach is its strict deterministic character. DTs do not have an error theory. As a result, they hide the imprecision that is often present in decision-making. The assumed, apparent precision in decision-making is attributed to the exactness of the categorisation of the condition states. This property may restrict a flexible decision-making, and induce a too rigid choice behaviour. Moreover, the degree of imprecision associated with possible decision outcomes is assumed non-existent and is left untouched. A solution to this problem was proposed in Witlox et al. (1997). The basic idea is to allow for gradual transition between condition states in the sense that fuzzy sets are introduced.

Another important issue concerns the validity of DT approach. A basic question in this respect, as also pointed by Timmermans and van der Heijden (1987, p. 301), is whether decision makers are able to report accurately on their decision-making process by means of a DT structure. In other words, are individuals capable of articulating the heuristic decision rules incorporated in a DT and can they execute the information-processing rules in a predeterminated sequence? In addition, there may exist the problem that individuals may mention too many attributes merely because they are aware of being observed; on the other hand, they may not name attributes that they consider selfevident. The problem here is that of "reactivity". How will the decision makers react if asked to describe their choice process? Will they demonstrate an elaborate, detailed, and well-organized decision-making? Or will they exhibit a rather unconscious, indifferent, and diverse decision-making? To some extent, the use of subtables can help to structure a decision problem. However, the point is that a DT structure will have to be validated ex post by the decision makers.

\section{Conclusions}

In summary, the main advantage of the decision table formalism is primarily its ability to easily check the consistency and completeness of the representation. Other formalisms such as decision trees, and production systems "if...then" rules are much more dependent upon a formal checking by the researcher and revision after the knowledge acquisition session (Goetgeluk et al., 1994). In 
contrast, the decision table can be used as a leading principle during such sessions, resulting in more reliable decision structures.

Given its property, the decision table formalism has a rich potential in spatial analysis in at least three areas of research. First, it can be used as a qualitative tool to elicit location factors and the complex structure of interdependencies among location factors in site selection. This was the topic of the present study. Compared to other more common methodologies many checks are built into the decision table formalism. Secondly, the decision table formalism may be used as a modelling tool. Locational decision making of firms could be simulated by activating condition states, which would lead to actions (locational decisions). In this context, its logic-based structure offers a richer potential than the commonly used algebraic models to incorporate veto criteria and non-compensatory decision making principles. Finally, the set of decision tables constitutes an expert system in its own right, or one that could be linked to geographic information systems. In other words, DTs can be used to implement a policy that determines specific actions.

We intend to further explore these options in our future publications. In doing so, some issues need to be addressed. First, there is hardly any research on the validity of the decision table formalism. In our discussion, we have identified some potential concerns, but of course the validity of the formalism can and should be empirically tested in future research. Secondly, the deterministic nature of the decision table formalism may be rather rigid in some applications. Hence, the issue of how to incorporate measurement error and intransitive rules should be high on the research agenda. Finally, it may also be very valuable to explore the possibilities of deriving decision tables from empirical data as opposed to knowledge elicitation sessions.

\section{References}

Arentze T A, Borgers A W J and Timmermans H J P, 1995, "The integration of expert knowledge in decision support systems for facility location planning" Computers, Environment and Urban Systems 19 227-247. 
Arentze T A, Borgers A W J and Timmermans H J P, 1996, "An efficient search strategy for siteselection decisions in an expert system" Geographical Analysis 28 126-146.

Chapman K, 1991, The International Petrochemical Industry: Evolution and Location. Oxford and Cambridge (Mass.), Basil Blackwell.

Codasyl, 1982, A Modern Appraisal of Decision Tables. Report of the Decision Table Task Group of the Conference on Data Systems Languages (CODASYL). New York, Association for Computing Machinery (ACM).

Goetgeluk R W, Goethals A, Oskamp A and Timmermans H J P, 1994, "Editorial Seminar on Choice Modelling in Housing Market Simulations" Netherlands Journal of Housing and the Built Environment 9 209-214.

Havenbedrijf Antwerpen, 1996, The Establishment of Industries in the Antwerp Port Zone. Antwerpen, Havenbedrijf Antwerpen.

Lucardie G L, 1994, Functional Object-Types as a Foundation of Complex Knowledge-Based Systems. Rijswijk, TNO Bouw, Ph.D. thesis.

McDaniel H Ed., 1970, Applications of Decision Tables: A Reader. Princeton, Brandon-Systems Press.

Molle W and Wever E, 1984, Oil Refineries and Petrochemical Industries in Western Europe. Aldershot, Gower Publishing Company.

Montalbano M, 1974, Decision Tables. Chicago, Scientific Research Associates.

Nguyen T A, Perkins W A, Laffey T J and Pecora D, 1985, "Checking an expert system knowledge base for consistency and completeness". In Proceedings of the $9^{\text {th }}$ International Joint Conference on Artificial Intelligence, NIJCAI (1985). Los Angeles, NIJCAI, 18-23 August $1985,375-378$

Pawlak, Z, Grzymala-Busse J W, Slowinski, R and Ziarko, W, 1995, "Rough sets" Comm. Of the ACM 38 89-95.

Preece A D and Shinghal R, 1994, "Foundation and application of knowledge base verification" International Journal of Intelligent Systems 9 683-701. 
Reilly K D, Salah A and Yang C.-C, 1987, "A logic programming perspective on decision table theory and practice" Data \& Knowledge Engineering 2 191-212.

Timmermans H J P and Heijden R E C M van der, 1987, "Uncovering spatial decision-making processes: a decision net approach applied to recreation choice behaviour" Tijdschrift voor Economische en Sociale Geografie LXXVIII 297-304.

Turban E, 1995, Decision Support and Expert Systems. Management Support Systems (4 ${ }^{\text {th }}$ Edition). Englewood Cliffs (New Jersey), Prentice-Hall.

Vanthienen J, 1986, Automatiseringsaspecten van de Specificatie, Constructie and Manipulatie van Beslissingstabellen. Leuven, Katholieke Universiteit Leuven, Departement Toegepaste Economische Wetenschappen, Ph.D. thesis, Nr. 60.

Vanthienen J and Dries E, 1992, "Developments in decision tables: evolution, application and a proposed standard". Research Report. No. 9227. Leuven, Katholieke Universiteit Leuven, Departement Toegepaste Economische Wetenschappen.

Vanthienen J and Dries E, 1994, "Illustration of a decision table tool for specifying and implementing knowledge based systems" International Journal on Artificial Intelligence Tools 3 267-288.

Vanthienen J and Wets G, 1994, "From decision tables to expert system shells" Data \& Knowledge Engineering 13 265-282.

Vanthienen J, Mues C, Aerts A and Wets G, 1995, "A modelling approach to KBS verification". In Ayel M and Rousset M.-C Eds. (1995) Proceedings of the European Symposium on the Validation and Verification of Knowledge Based-Systems - EUROVAV-95: June 26-28, 1995. Chambéry (St. Baldoph), EUROVAV-95, 155-171.

Verhelst M, 1980, De Praktijk van Beslissingstabellen. Deventer and Antwerp, Kluwer.

Witlox F, 1998, Modelling Site Selection: A Relational Matching Approach based on Fuzzy Decision Tables. Eindhoven, Technische Universiteit Eindhoven, Ph.D. thesis.

Witlox F, Arentze T A and Timmermans H J P, 1997, "Constructing and consulting fuzzy decision tables". In Timmermans H J P Ed. (1997) Decision Support Systems in Urban Planning. London, E \& FN Spon, 156-174. 
Zadeh, L, 1965, “Fuzzy sets” Information and Control 8 338-353. 